\title{
Heat Transfer Measurement and Modeling in Rigid High- Temperature Reusable Surface Insulation Tiles
}

\author{
Kamran Daryabeigi, ${ }^{*}$ Jeffrey R. Knutson, ${ }^{\dagger}$ \\ NASA Langley Research Center, Hampton, Virginia, 23681 \\ and \\ George R. Cunnington \\ Cunnington and Associates, Palo Alto, California, 94303
}

\begin{abstract}
Heat transfer in rigid reusable surface insulations was investigated. Steady-state thermal conductivity measurements in a vacuum were used to determine the combined contribution of radiation and solid conduction components of heat transfer. Thermal conductivity measurements at higher pressures were then used to estimate the effective insulation characteristic length for gas conduction modeling. The thermal conductivity of the insulation can then be estimated at any temperature and pressure in any gaseous media. The methodology was validated by comparing estimated thermal conductivities with published data on a rigid high-temperature silica reusable surface insulation tile. The methodology was also applied to the alumina enhanced thermal barrier tiles. Thermal contact resistance for thermal conductivity measurements on rigid tiles was also investigated. A technique was developed to effectively eliminate thermal contact resistance on the rigid tile's cold-side surface for the thermal conductivity measurements.
\end{abstract}

\section{Nomenclature}

$=$ coefficients

$=$ specific heat, $\mathrm{J} /(\mathrm{kg} \cdot \mathrm{K})$

$=$ thermal conductivity, $\mathrm{W} /(\mathrm{m} . \mathrm{K})$

$=$ effective thermal conductivity, $\mathrm{W} /(\mathrm{m} . \mathrm{K})$

$=$ insulation thickness, $\mathrm{m}$

$=$ characteristic length, $\mathrm{m}$

$=$ pressure, torr $(\mathrm{mm}$ of $\mathrm{Hg})$

$=$ Prandtl number

$=$ heat flux, $\mathrm{W} / \mathrm{m}^{2}$

$=$ thermal contact resistance, $\mathrm{K} \cdot \mathrm{m}^{2} / \mathrm{W}$

$=$ temperature, $\mathrm{K}$

$=$ time, $\mathrm{s}$

$=$ spatial coordinate, $\mathrm{m}$

$=$ thermal accommodation coefficient

$=$ specific heat ratio

$=$ molecular mean free path, $\mathrm{m}$

$=$ density, $\mathrm{kg} / \mathrm{m}^{3}$

Subscripts

$B \quad=$ sample cold side

C = test setup cold side (water-cooled plate)

\footnotetext{
* Aerospace Engineer, Structural Mechanics and Concepts Branch, Mail Stop 190. Senior Member, AIAA.

${ }^{\dagger}$ Electrical Engineering Technician, Systems Integration and Test Branch, Mail Stop 424.

* Owner, Cunnington and Associates. Associate Fellow, AIAA.
} 


$\begin{array}{ll}g & =\text { gas } \\ H & =\text { sample hot side } \\ r & =\text { radiation } \\ s & =\text { solid conduction } \\ s r & =\text { combined solid conduction and radiation }\end{array}$

\section{Acronyms}

$\begin{array}{ll}\text { AETB } & =\text { alumina enhanced thermal barrier } \\ \mathrm{LI} & =\text { Lockheed insulation } \\ \mathrm{TRSH} & =\text { transient radiant step heating }\end{array}$

\section{Introduction}

$\mathrm{H}$ eat transfer through fibrous insulations has been the subject of great interest in the aerospace community because of the use of fibrous insulations in thermal protection systems (TPS). The fibrous insulation systems used are either in flexible or rigid form. The advanced flexible reusable surface insulation (AFRSI) blankets and the rigidized reusable surface insulation tiles on the Space Shuttle Orbiter are examples of flexible and rigid fibrous insulations, respectively. Higher-fidelity modeling was previously used for modeling heat transfer in various flexible fibrous insulation systems. ${ }^{1}$ The focus of the present work is on modeling heat transfer in rigid fibrous insulations.

The standard practice for modeling heat transfer in fibrous insulations uses thermal conductivity measurements as a function of temperature and pressure obtained using either steady-state ${ }^{2}$ or transient ${ }^{3}$ test techniques. The tabulated thermal conductivity data are then used for analysis and design of TPS. One shortcoming of this technique is that the generated thermal conductivity data are applicable only to the specific gaseous test environment. Using the methodology proposed here, limited number of thermal conductivity data generated in one gaseous environment can be used to generate thermal conductivity data in various gaseous environments.

Heat transfer through these high-porosity rigid insulations is composed of combined radiation and conduction heat transfer. Conduction consists of both solid and gaseous conduction. The overall heat transfer modeling for rigid and flexible insulations is similar, but the relative magnitude of various modes of heat transfer varies between the two. Solid conduction is a significant component of heat transfer in rigid insulations, with its significance increasing with increasing insulation density. Radiation's significance increases with increasing temperature, and decreases with increasing insulation density. Gas conduction is negligible in a vacuum, and increases with increasing static pressure and temperature. Theoretical modeling of solid conduction through fibers and points of contact between them is difficult, therefore, various empirical and semi-empirical relations have traditionally been used. ${ }^{4,5}$ Modeling of gas conduction in fibrous insulations requires knowledge of characteristic length (pore size) and gas mean free path, ${ }^{6,7}$ and is well established. Modeling of radiation heat transfer through fibrous insulations is more complicated and has been the subject of numerous studies, with a comprehensive review of various radiation models provided elsewhere. ${ }^{8}$ The simplest approach has been to use a combined radiation and conduction heat transfer model with radiation conductivity in terms of a Rosseland mean coefficient based on the diffusion approximation. ${ }^{9,10}$ This approach is used in the present study. The most comprehensive radiation model contains a rigorous formulation for scattering properties of fibrous insulations that accounts for the two-dimensional scattering characteristics of fibers, with the resulting radiation model using deterministic parameters that define the composition and morphology of the medium: distributions of fiber size and orientation, fiber volume fraction, and the spectral complex refractive index of the fibers. ${ }^{11,12}$ This comprehensive radiation model is limited to the independent scattering regime, requiring detailed knowledge of fiber orientation with respect to the boundaries, and accurate spectral complex refractive index data. This formulation has been successfully developed and validated for the Lockheed insulation LI-900 rigid silica tile at a nominal density of $144 \mathrm{~kg} / \mathrm{m}^{3}\left(9 \mathrm{lb} / \mathrm{ft}^{3}\right)$ used on the Space Shuttle Orbiter. ${ }^{5}$

The main purpose of the present work is to investigate whether limited thermal test data can be used to develop accurate heat transfer models in various rigid fibrous insulation tiles. For insulations with single fiber composition, whether flexible or rigid, the individual contributions of the radiation and solid conduction heat transfer can be inferred from experimental data in conjunction with the appropriate thermal model. ${ }^{1,5}$ For insulations with multiple fiber compositions such as alumina enhanced thermal barrier (AETB) tiles which consist of silica, alumina, and aluminoborosilicate fibers, the estimation of the individual contributions of the radiation and solid conduction components of heat transfer is more complicated. Therefore, the methodology used here consists of estimation of 
the combined contribution of solid conduction and radiation heat transfer modes (without breaking it into its individual components) from thermal conductivity data in a vacuum ( 0.001 torr) at various temperatures between 300 and $1400 \mathrm{~K}$. The gas conduction characteristic length is then estimated from measured thermal conductivity data at higher pressures to obtain the gas conduction model.

For flexible insulations, the flexibility of the insulation and negligible solid conduction mode of heat transfer in the insulation result in negligible thermal contact resistance at the boundaries of test samples in the experimental setup used for measuring thermal performance. For rigid insulations, the solid conduction mode of heat transfer is significant and obtaining perfect flatness on sample and test setup surfaces is not easily achievable. Even a sample with a flat surface may bow from thermal deformations during testing, therefore, thermal contact resistance at the boundaries of the test sample may be significant. For the thermal test setup used in the present study with large temperature differences maintained across the sample thickness, thermal contact resistance on the sample hot side is ignored. On the sample hot side the main mode of heat transfer is radiation with a small component of solid conduction. Since thermal contact resistance only affects the solid conduction mode, thermal contact resistance on the sample hot side can be ignored. Conversely on the sample cold side, the main mode of heat transfer is solid conduction, therefore, thermal contact resistance can be significant, which manifests itself as radiation and gas conduction in the void spaces between the bottom of the test sample and the top of the test setup cold-side surface. In most thermal conductivity measurement techniques, compressive forces are applied on the boundaries of test samples in order to minimize thermal contact resistances at the boundaries. In the case of the test setup used in this study, it was found that application of a compressive load of $480 \mathrm{~N} / \mathrm{m}^{2}\left(10 \mathrm{lb} / \mathrm{ft}^{2}\right)$ was not sufficient to eliminate thermal contact resistance, mainly because the rigid test sample bows when it has a thermal gradient through its thickness. In order to account for thermal contact resistance on the sample cold side, flush mounted thermocouples were installed on the sample cold-side surface, and the corresponding temperature differences between this surface and the top of the test setup cold-side surface were measured. Furthermore, a novel technique was developed in order to effectively eliminate thermal contact resistance on the sample cold side, by utilizing a thin layer of liquid bismuth alloy between the sample and test setup cold-side surfaces.

The first objective of the present study is to investigate whether thermal contact resistance can be significant for thermal measurements on rigid insulation tiles, and if so, investigate a technique for eliminating the thermal contact resistance. The second objective is to investigate a methodology for estimating thermal conductivity of rigid insulations in various gaseous environments for temperature range of $300-1400 \mathrm{~K}$ and pressure range of 0.001 to 760 torr, based on a limited set of thermal conductivity data in one gas. The validity of this methodology is investigated by comparison with published data on the LI-900 tile. The methodology is also applied to AETB-8 and AETB-12 tiles.

\section{Experimental Approach}

Data reported here were generated using the steady-state thermal test setup at NASA Langley Research Center (LaRC) which provides effective thermal conductivity data with large temperature gradients maintained across the sample thickness. A limited set of thermal conductivity data was also generated using the transient radiant step heating technique for validation of heat transfer models. The test samples and the measurement techniques are briefly described.

\section{A. Test Samples}

The LI-900 tile used is composed of silica fibers at a density of $141 \mathrm{~kg} / \mathrm{m}^{3}$. This is the most extensively characterized rigid

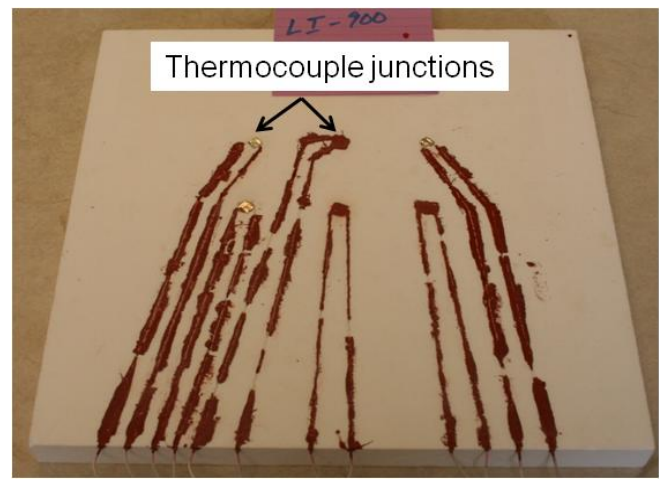

a)

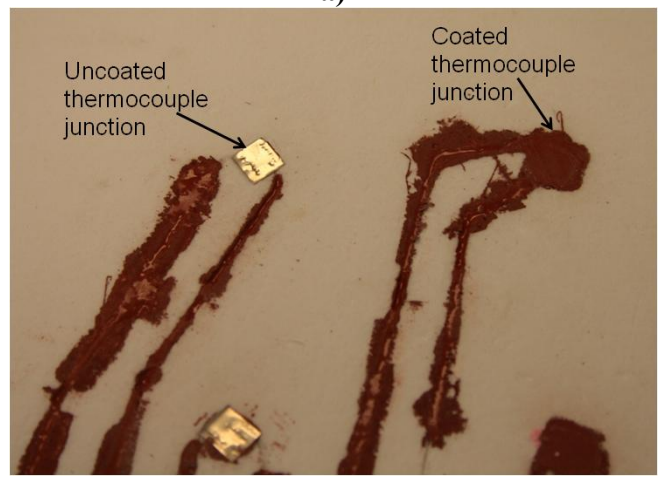

b)

Figure 1. Photographs of the LI-900 tile cold-side surface instrumented with flush mounted thermocouples: a) entire sample; b) close-up showing coated and uncoated thermocouple junctions. 
insulation $^{2,5}$ and is used on the Space Shuttle Orbiter. The mean fiber diameter for the sample used in this study was $1.9 \mu \mathrm{m}$ with a standard deviation of $1 \mu \mathrm{m}$. Data from this tile were used for validation of experimental and modeling methodology. Two AETB tiles ${ }^{\S}$ were also tested: AETB-8 and AETB-12 tiles with densities of 134 and $188 \mathrm{~kg} / \mathrm{m}^{3}$ (nominal densities of 8 and $12 \mathrm{lb} / \mathrm{ft}^{3}$ ), respectively. The AETB tiles used in this study have nominal composition of $68 \%$ silica, $20 \%$ alumina, and $12 \%$ aluminoborosilicate fibers. AETB tiles by other manufacturers may contain up to $3 \%$ silicon carbide powder which may result in lower thermal conductivities at higher temperatures compared to the samples studied in the present study, as had previously been observed ${ }^{13}$ on the LI2200 silica tile with a nominal density of $352 \mathrm{~kg} / \mathrm{m}^{3}\left(22 \mathrm{lb} / \mathrm{ft}^{3}\right)$. All three samples used in this study were $304.8 \times$ $304.8 \mathrm{~mm}, 25.4 \mathrm{~mm}$ thick. The cold side of the rigid samples was instrumented with 3 to 6 flush mounted thermocouples located within the central $100 \times 100 \mathrm{~mm}$ of the sample center to provide the average sample coldside temperature. A photograph of the cold-side surface of the LI-900 sample is provided in Fig. 1a showing six flush mounted thin-foil thermocouples. Each thermocouple was fabricated by welding $0.127-\mathrm{mm}$ diameter type K thermocouple wires to a $0.127-\mathrm{mm}$ thick, $12.5-\mathrm{mm}$ square stainless steel foil, with the thin foil serving as the effective thermocouple junction. The foil thermocouples were typically coated with a thin layer of room temperature vulcanizing silicone (RTV). For the LI-900 sample, three of the foil thermocouples were coated with RTV, and three thermocouples were uncoated. A close-up photograph showing the coated and uncoated thermocouple junctions is shown in Fig. 1b. Small trenches were made in the test sample surface in order to bury the thermocouple lead wires. The trenches were then covered with RTV.

\section{B. Effective Thermal Conductivity Measurements}

Steady-state effective thermal conductivity data with large temperature differences maintained across the sample thickness were generated in the LaRC thermal-vacuum testing apparatus, described in detail elsewhere. ${ }^{14,15} \mathrm{~A}$ schematic of the test setup is shown in Fig. 2. The main components are: a quartz lamp radiant heater array, an Inconel septum plate, a water-cooled plate, test sample, and refractory ceramic board insulation. The planar area of the water-cooled and septum plates is $304.8 \times 304.8 \mathrm{~mm}$. The insulation sample $(304.8 \times 304.8 \times 25.4 \mathrm{~mm})$ is placed between the water-cooled and septum plates. Use of a test sample with such a large ratio of planar dimension to thickness ensures one dimensional heat transfer in the test setup, especially in the central region of the test assembly. The entire assembly is then surrounded by refractory ceramic insulation. The water-cooled plate is equipped with

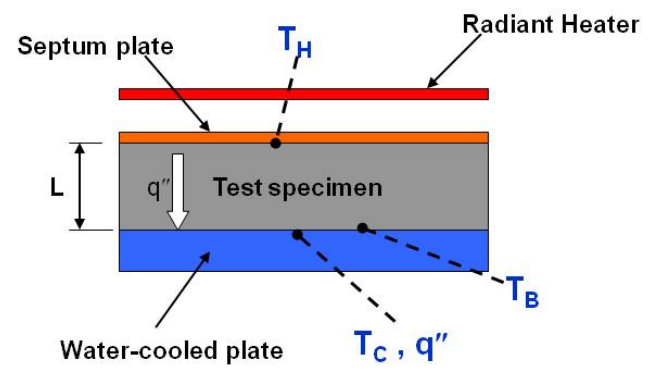

Figure 2. Schematic of steady-state test setup. nine flush-mounted thin-film heat flux gages that provide simultaneous heat flux and temperature measurements, while the septum plate is instrumented with 23 metal-sheathed Type K thermocouples. Data from instrumentation located in the central $127 \times 127 \mathrm{~mm}$ section of the test setup on the water-cooled and septum plates are used for the effective thermal conductivity measurements, while data from instrumentation located outside of the central region are used to assess deviations from one dimensional heat transfer in the setup. The average heat flux and temperature calculated from five heat flux gages located in the central region of the water-cooled plate provide the heat flux, $q^{\prime \prime}$, and test setup cold-side temperature, $T_{C}$, while the average temperature calculated from 13 thermocouples located in the central region of the septum plate provides the hot-side temperature, $T_{H}$. The cold side of the rigid test samples was instrumented with 3 to 6 flush mounted thin foil thermocouples located within the central $100 \times 100 \mathrm{~mm}$ of the sample center to provide the average sample cold-side temperature, $T_{B}$. In the absence of thermal contact resistance on the sample cold side, $T_{B}$ and $T_{C}$ should be equal. The effective thermal conductivity is then calculated from

$$
k_{e}=\frac{q^{\prime \prime} L}{\left(T_{H}-T_{B}\right)}
$$

For an optically thick insulation, the effective thermal conductivity is related to thermal conductivity through

$$
k_{e}=\frac{1}{T_{H}-T_{B}} \int_{T_{B}}^{T_{H}} k \partial T
$$

\footnotetext{
${ }^{\S}$ Forrest Machining Inc
} 
Even though the test setup does not directly yield thermal conductivity, the measured effective thermal conductivities can be used to estimate thermal conductivity from Eq. (2).

Tests were conducted with various $T_{H}$ between 530 and $1360 \mathrm{~K}$. At each $T_{H}$ set point, tests were conducted at various static pressures between 0.001 and 750 torr in nitrogen gas. For nominal $T_{H}$ of $533,811,1089$, and $1366 \mathrm{~K}$, data were generated at nominal pressures of $0.001,0.1,1,10,100$, and 750 torr. For nominal $T_{H}$ of 672,950 , and $1228 \mathrm{~K}$, data were generated at a nominal pressure of 0.001 torr only. A detailed uncertainty analysis was conducted for each measured quantity: hot- and cold-side temperatures, and heat fluxes. The overall uncertainty consisted of the contributions of random and bias uncertainties for each measured quantity, and uncertainties due to spatial non-uniformity of spatially-averaged quantities.

\section{Modification of Effective Thermal Conductivity Setup to Eliminate Thermal Contact Resistance}

The effective thermal conductivity test setup was modified in order to eliminate thermal contact resistance on the sample cold-side surface. The modification consists of using a thin layer of liquid bismuth alloy located between the top of the water-cooled plate and the bottom of the rigid insulation test sample and within a containment structure. Bismuth alloy is a high thermal conductivity metal that melts at approximately $47^{\circ} \mathrm{C}$. A quantity of solid bismuth alloy is placed on top of the water-cooled plate, as shown in Fig. 3. A containment structure consisting of angle sheet metal measuring $25.4 \times 12.7 \times 1.6 \mathrm{~mm}$ thick is attached to the water-cooled plate forming a perimeter around the water-cooled plate having dimensions of $330 \times 330$ $\mathrm{mm}$, as seen in Fig. 3. Water controlled to approximately $54^{\circ} \mathrm{C}$ is circulated through the water-cooled plate thus causing the solid bismuth alloy to melt and form a liquid layer. The insulation test sample is then laid upon the liquid metal and is centered over the water-cooled plate as seen in Fig. 4. The septum plate is then installed on the test sample as seen in Fig. 5. This causes the test sample to sink into the liquid bismuth alloy until it touches the $1.6 \mathrm{~mm}$ thick containment structure, thus displacing all the liquid bismuth alloy except a $1.6 \mathrm{~mm}$ thick layer which is trapped between the test sample and the water-cooled plate. Because the containment structure is larger than the water-cooled plate and the test sample, there is a 12.7 $\mathrm{mm}$ gap around the perimeter of the test sample which serves as an accumulation area for the displaced liquid bismuth alloy. Within this accumulation area the bismuth rises to a height of $6.3 \mathrm{~mm}$. There is a spout built into the containment structure 6.3 $\mathrm{mm}$ above the level of the water-cooled plate, as shown in Fig. 4, for draining off any excess liquid bismuth alloy that is displaced upon insertion of the test sample. A rigid test sample can bow due to thermal expansion of the hot side during an

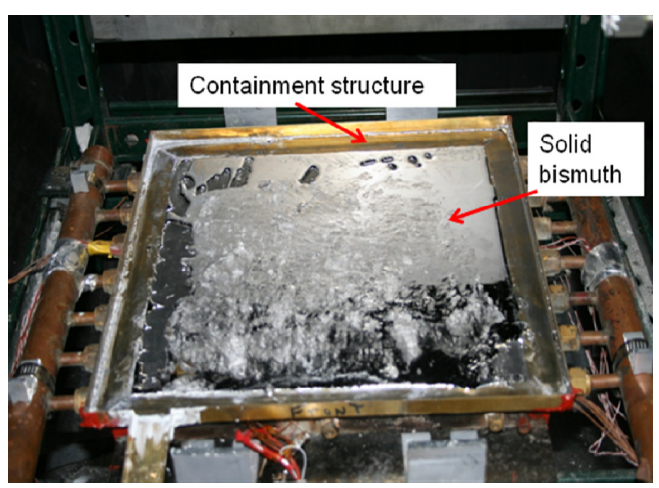

Figure 3. Photograph of water-cooled plate with bismuth alloy partially covering it.

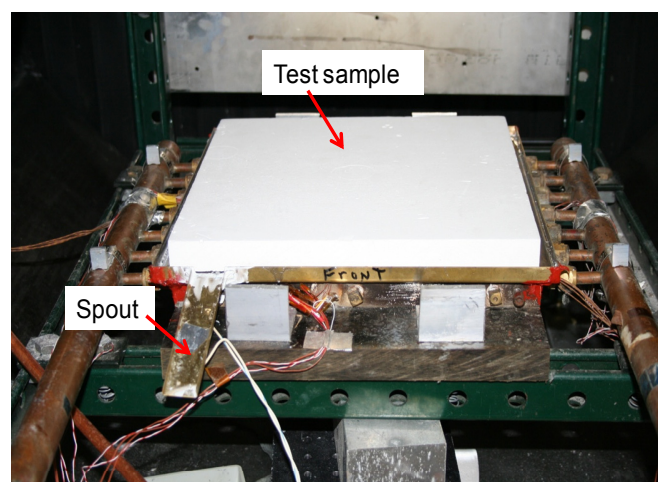

Figure 4. Photograph of rigid tile test sample in test setup.

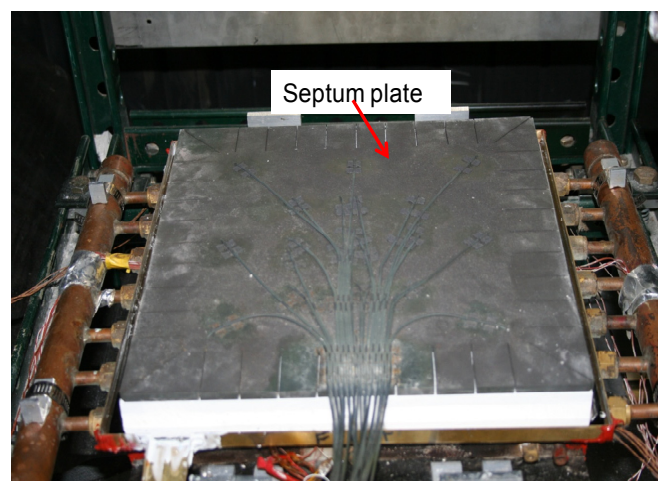

Figure 5. Photograph of septum plate set on rigid tile test sample in test setup. effective thermal conductivity test causing a concave area to form at the bottom of the sample. When this happens liquid bismuth alloy is free to flow from the accumulation area to fill the newly created concave area at the sample cold-side surface, thus maintaining full contact between the bismuth and the bottom of the test sample regardless of dynamic warping during the testing process. The presence of the relatively high thermal conductivity liquid bismuth alloy layer between the bottom of the test sample and the top of the water-cooled plate effectively eliminates the thermal contact resistance. The measured effective thermal conductivity and predicted thermal conductivity data using the modified test setup will be referred to as the "present technique." 


\section{Thermal Conductivity Measurements}

As part of this study standard thermal conductivity data were measured at a thermophysical properties laboratory using the transient radiant step heating (TRSH) technique referred to as the "three point method", described in detail elsewhere. ${ }^{16}$ This measurement technique was used to generate thermal conductivity data on AETB samples in the temperature range of $290-1173 \mathrm{~K}$ at various static pressures in nitrogen gas for validation of the thermal conductivity data estimated from effective thermal conductivity measurements. The reported uncertainty of these measurements was $\pm 10 \%$.

\section{Analytical Approach}

The governing one-dimensional conservation of energy equation for the problem of combined radiation and conduction in an optically thick medium is given by

$$
\rho c \frac{\partial T}{\partial t}=\frac{\partial}{\partial y}\left(k \frac{\partial T}{\partial y}\right)
$$

where $k$ is obtained by superposition of the thermal conductivities due to solid conduction, gas conduction, and radiation $^{1,5}$

$$
k=k_{s}+k_{g}+k_{r}
$$

The general formulation for each of the thermal conductivity terms for various flexible and rigid fibrous insulation samples is discussed in detail elsewhere. ${ }^{1,5}$ As discussed previously, estimation of the individual components of solid conduction and radiation heat transfer in multi-fiber compositions is rather complicated. Therefore, the combined contribution of solid conduction and radiation modes of heat transfer was obtained from the experimental effective thermal conductivity data at 0.001 torr, where gas conduction is insignificant. For fibrous insulations, the solid conduction component of thermal conductivity varies almost linearly with temperature, while the radiation component of thermal conductivity varies with temperature to the third power. Therefore, it was assumed that the thermal conductivity of the test sample at this low pressure ( 0.001 torr) was a third order polynomial function of temperature

$$
k_{s r}=k_{s}+k_{r}=\sum_{i=0}^{3} a_{i} T^{i}
$$

The effective thermal conductivity measurements at 0.001 torr were used in conjunction with Eqs. (2) and (5) to obtain the unknown coefficients, $a_{i}$. Once $k_{s r}$ is known, all that is needed is the gas conduction characteristic length to complete the heat transfer modeling. The gas thermal conductivity is given by ${ }^{6}$

$$
k_{g}(T, P)=\frac{k_{g 0}(T)}{1+2 \frac{\beta(T)}{\operatorname{Pr}(T)} \frac{\lambda(T, P)}{L_{C}}}
$$

where $k_{g 0}(T)$ is the thermal conductivity of the gas at atmospheric pressure, $P r$ is the Prandtl number, and $\lambda$ is the gas mean free path. The parameter $\beta$ is defined as

$$
\beta(T)=\left(\frac{2-\alpha}{\alpha}\right) \frac{2 \gamma(T)}{(\gamma(T)+1)}
$$

Since there is limited data for the thermal accommodation coefficient, $\alpha$, between various gases and fiber materials, a thermal accommodation coefficient of unity was used. Once $k_{g 0}(T), \operatorname{Pr}(T), \lambda(T, P)$, and $\gamma(T)$ are known from the literature, ${ }^{17,18,19}$ the gas conduction contribution in fibrous insulations can be easily calculated if the gas conduction characteristic length $\left(L_{C}\right)$ is known. $L_{C}$ is a measure of insulation pore size and is independent of the gaseous medium used. Experimental effective thermal conductivity data at higher pressures were used to estimate $L_{C}$. The thermal conductivity was calculated by superposition of radiation and solid conduction thermal conductivity from Eq. (5) and gas conduction thermal conductivity from Eq. (6), integrating the resulting thermal conductivity according to Eq. (2) to get the predicted effective thermal conductivity. The objective was to find a gas conduction characteristic length that would minimize the sum of the squares of the differences between measured and predicted effective thermal conductivities at higher pressures. 


\section{Discussion of Results}

\section{A. Thermal Contact Resistance}

Thermal contact resistance was investigated for the tests on the LI-900 tile. Data were first generated without using the liquid bismuth alloy layer for eliminating thermal contact resistance. For these tests only, test pressures were $0.001,0.076,0.76,7.6,76$, and 750 torr instead of the standard nominal test pressures discussed previously. The average water-cooled plate temperature, $T_{C}$, for all the tests was $298.3 \pm 2.6 \mathrm{~K}$. The LI-900 tile had 6 flush mounted thin foil thermocouples in the central $100 \times 100 \mathrm{~mm}$ region of its cold-side surface. Three thermocouples were uncoated, while three were coated with a thin layer of RTV. The variation of the difference between measured sample cold-side temperature, $T_{B}$, for both the uncoated and coated thermocouples, and water-cooled plate temperature, $T_{C}$, as a function of sample hot-side temperature, $T_{H}$, at a static pressure of 0.001 torr is shown in Fig. 6. There was a significant temperature difference between the sample and water-cooled plate which increased with increasing $T_{H}$, signifying presence of thermal contact resistance, with the data from uncoated thermocouples providing temperature differences that were 2 to 2.6 times higher compared to coated thermocouples. The temperature differences, $T_{B}-T_{C}$, varied between 12.7 and $90.7 \mathrm{~K}$ for coated thermocouples, and between 25.9 and $201.5 \mathrm{~K}$ for uncoated thermocouples. The corresponding standard deviations for the average calculated $T_{B}$ from the three thermocouples at each value of $T_{H}$, representing the spatial non-uniformity of thermocouples, varied between 5.3 and $16.9 \mathrm{~K}$ for coated thermocouples, and between 6.4 and $17.7 \mathrm{~K}$ for uncoated thermocouples, and are shown as the error bars in Fig. 6. At this low pressure the only mode of heat transfer in the void spaces between sample cold-side surface and water-cooled plate was radiation heat transfer. The uncoated thermocouples had a lower emittance compared to the coated thermocouple, thus, did not exchange heat with the water-cooled plate as efficiently as the coated ones, resulting in local hot spots. The coated thermocouples exchanged heat with the water-cooled plate more efficiently due to their higher emittance, and therefore, reached temperatures that were closer to the water-cooled plate temperatures compared to the uncoated thermocouples.

The variation of temperature difference, $T_{B}-T_{C}$, for the uncoated thermocouples as a function of static pressure for various $T_{H}$ is shown in Fig. 7. The temperature difference increased with increasing $T_{H}$ (increasing heat flux), but decreased with increasing static pressure. The same trend was observed with coated thermocouple data. The main modes of heat transfer in the void spaces between the bottom of the test sample and the top of the water-cooled plate were radiation and gas conduction. At low pressures, gas conduction was negligible, thus resulting in larger temperature differences in the void spaces. As chamber gas pressure increased, gas conduction increased, resulting in more efficient heat exchange in the void spaces which resulted in the temperature of the sample cold side being closer to the temperature of the water-cooled plate. The temperature difference generally decreased with increasing pressure for $P \leq 7.6$ torr. The temperature differences approached a minimum at 7.6 torr, followed by a slight increase at higher pressures as seen for the data at higher $T_{H}$ in the figure. For $P \geq 7.6$ torr, the average temperature differences were 3.6 and $37 \mathrm{~K}$ for $\mathrm{T}_{\mathrm{H}}$ of 533 and $1366 \mathrm{~K}$, respectively.

Thermal contact resistance was calculated using the average heat flux measured in the central region of test setup, $q^{\prime \prime}$, and the corresponding temperature differences between the test sample cold side and water-cooled plate for both uncoated and coated thermocouples. The variation of thermal contact resistance with test chamber static pressure for various $T_{H}$ is shown in Fig. 8 for the data from uncoated thermocouples. Thermal contact resistance 
decreased with increasing pressure from 0.001 to 7.6 torr, and then approached a constant value after 7.6 torr. Thermal contact resistance was generally insensitive to $T_{H}$, except at 0.001 torr, where contact resistance increased with decreasing $T_{H}$. The thermal contact resistances at all test temperatures and pressures varied between 0.007 and 0.152 , and between 0.005 and 0.074 $\mathrm{K} . \mathrm{m}^{2} / \mathrm{W}$ for uncoated and coated thermocouples, respectively. These data show that thermal contact resistance exists at the rigid tile's boundaries and that it becomes very significant at lower static pressures, despite applying a compressive load of 480 $\mathrm{N} / \mathrm{m}^{2}$ on the tile. Most steady-state thermal conductivity measurement techniques rely on the application of compressive loads to minimize and/or eliminate thermal contact resistance, but it appears that the application of compressive load may not be sufficient. It is recommended that flush mounted thermal instrumentation be used on the surfaces of test samples to determine the magnitude of thermal contact resistance, and use temperature data from these instrumentation instead of thermal instrumentation embedded in test setup to perform the required analysis for thermal conductivity calculations.

Tests on the LI-900 tile were repeated with liquid bismuth alloy used between the water-cooled plate and the test sample cold side. Water for the water-cooled plate was heated in order to cause melting of the bismuth alloy. The average water-cooled plate temperature, $T_{C}$, for these tests was $330 \pm 6.5 \mathrm{~K}$. There was insignificant temperature difference between the sample coated and uncoated thermocouples, with standard deviation for all 6 thermocouples ( 3 coated and 3 uncoated) for each $T_{H}$ varying between 0.2 and $2.7 \mathrm{~K}$. The variation of $T_{B}-T_{C}$ as a function of sample hot-side temperature at a static pressure of 0.001 torr is shown in Fig. 9. The corresponding data for the uncoated and coated thermocouples for the tests without the liquid bismuth alloy are also shown in the figure for comparison. Use of the liquid bismuth alloy resulted in significantly lower temperature differences that varied between 0.8 and $8.6 \mathrm{~K}$. These temperature differences were the normal temperature drops through the 1.6-mm thick liquid bismuth alloy layer due to conduction heat transfer. The temperature differences increased slightly with increasing test pressure due to the higher heat fluxes imposed. The thermal resistance between the sample cold side and water-cooled plate varied between 0.003 and $0.006 \mathrm{~K} . \mathrm{m}^{2} / \mathrm{W}$, which was the thermal resistance associated with heat transfer through the liquid bismuth layer. Therefore, it can be concluded that the use of the liquid bismuth alloy effectively eliminated thermal contact resistance between the test sample cold side and water-cooled plate. Furthermore, effective thermal conductivity data were generated with the test sample cold-side temperatures that were almost constant and not that significantly different from the water-cooled plate temperatures.

\section{B. LI-900}

Effective thermal conductivity data for the LI-900 tile at 0.001 torr calculated using different techniques are shown in Fig. 10. Data generated using liquid bismuth alloy are designated "present technique" and are shown using circular symbols with their associated measurement uncertainties. Three other sets of data calculated from the test without using liquid bismuth are also shown in the figure. The effective thermal conductivity calculated using the water-cooled plate temperature, $\mathrm{T}_{\mathrm{C}}$, in Eq. (1) is shown using square symbols and designated as "w/o bismuth$\mathrm{T}_{\mathrm{C}}$." The effective thermal conductivity data calculated using the average temperature for the three uncoated and three coated thermocouples on the sample cold-side surface are also shown in the figure. For hot-side temperatures up to $1100 \mathrm{~K}$, the differences between the different techniques were within the experimental uncertainty range of the "present technique" data, but at temperatures exceeding $1100 \mathrm{~K}$ data using $T_{C}$ and uncoated $T_{B}$ were outside the experimental uncertainty range of the "present technique" data. Some overall trends can be discerned from the data. 
The data using $T_{C}$ underestimated effective thermal conductivity because the thermal contact resistance between the sample and water-cooled plate was ignored. These effective thermal conductivities underestimated the "present technique" by 2.5 to $12 \%$ with a root mean square (rms) deviation of $8.3 \%$. The data using uncoated $T_{B}$ overestimated effective thermal conductivity data because of the associated relatively higher localized panel cold-side temperatures resulting from using the low-emittance foil thermocouple junctions. These effective thermal conductivities overestimated the "present technique" data by 7.2 to $11.9 \%$ with an rms deviation of $10.3 \%$. The data using coated $T_{B}$ almost matched the "present technique" results, with an rms deviation of $2.1 \%$. In summary, if thermal contact resistance on the panel cold-side surface is ignored and the measured temperatures from the water-cooled plate are used, erroneously low effective thermal conductivities are generated. On the other hand, if the panel cold side is instrumented with thermocouples, one may get erroneously high effective thermal conductivities depending on the emittance of the thermocouples.

Measured LI-900 effective thermal conductivity data using the present technique at pressures of $0.001,1$, and 10 , and 100 torr as a function of $T_{H}$ is shown in Fig. 11. These data were generated with liquid bismuth alloy layer used in the test setup. The symbols represent the measured data and the error bars represent the experimental uncertainties obtained from detailed uncertainty analysis for each data point. The average uncertainty for the effective thermal conductivity test data on the LI-900 tile for all test temperatures and pressures was $\pm 9.1 \%$. Average $T_{B}$ for these measurements at all test temperatures and pressures was $333.6 \pm 8.5 \mathrm{~K}$. The effective thermal conductivity data at 0.001 torr pressure were used in conjunction with Eqs. (2) and (5) for estimating $k_{s}$, the thermal conductivity of LI-900 in a vacuum which is comprised of the combined contribution of solid conduction and radiation heat transfer modes. The variation of predicted LI-900 thermal conductivity with temperature at 0.001 torr pressure is shown in Fig. 12 and is labeled as "present technique." The effective thermal conductivity data at 0.001 torr pressure from Fig. 10 using the water-cooled plate temperature for calculating the effective thermal conductivity data were also used to infer thermal conductivity, with the resulting predicted thermal conductivity shown in Fig. 12 and labeled as "w/o bismuth- $\mathrm{T}_{\mathrm{C}}$." The latter data generally underpredicted the "present technique" data with differences varying between $-25 \%$ and $5.4 \%$, with an $\mathrm{rms}$ deviation of $9.1 \%$. The historical LI-900 tile data ${ }^{2,5}$ are also shown in the figure, along with their reported uncertainty of $\pm 10 \%$. The historical data presented here correspond to a tile that had a mean silica fiber diameter of $1.7 \mu \mathrm{m}$ with standard deviation of $1.26 \mu \mathrm{m} .{ }^{5}$ The LI-900 sample used in this study had a mean fiber diameter of $1.9 \mu \mathrm{m}$ with a standard deviation of $1 \mu \mathrm{m}$. The smaller the diameter and the lower the standard deviation of fiber diameter, the lower the radiation component of heat transfer in the tile, and the lower the thermal conductivity. ${ }^{5}$ Therefore, it was expected that the thermal conductivity data on the present tile would be slightly different from the historical data because of the differences in fiber size distribution. However, the "present technique" data had good agreement with the historical data. The predicted thermal conductivity matched published data to within the published data's uncertainties at all temperatures except the $800-1000 \mathrm{~K}$ range. The rms deviation between predictions and published data at this pressure was $12.3 \%$. The close agreement indicates that the experimental setup incorporating 
liquid bismuth alloy layer and the analysis methodology for estimating thermal conductivity from effective thermal conductivity measurements provides accurate modeling of thermal conductivity of LI-900 tile in a vacuum.

The gas conduction characteristic length (pore size) was determined to be $7.72 \times 10^{-5} \mathrm{~m}$ from the effective thermal conductivity measurements in nitrogen at higher pressures based on the procedure described previously. Using this characteristic length, then gas thermal conductivities were generated at various pressures and temperatures according to Eq. (6), and added to the combined contribution of solid conduction and radiation thermal conductivity, to calculate thermal conductivity of LI-900 at various pressures and temperatures in air. The comparison of predicted thermal conductivities with published data $^{2,5}$ in air is shown in Fig. 13a for static pressures of 0.076 and 76 torr, and in Fig. 13b for static pressures of 7.6 and 760 torr. The solid lines represent predicted data using the present technique, while the symbols represent published data. The reported $\pm 10 \%$ uncertainties for the published data are also included in the figures. There is good overall agreement between the predictions and published data at all pressures. The rms deviations between measurements and published data were $13.6,9.7,10.3,6.4$, and $10.6 \%$ at pressures of $0.076,0.76,7.6$, 76 , and 760 torr, respectively. The overall rms deviation for data at all pressures was $10.5 \%$. The close agreement between predictions and published data validates the overall testing and modeling methodology used in the present study.

\section{AETB-8}

The AETB- 8 tile had a density of $133.6 \mathrm{~kg} / \mathrm{m}^{3}$ and was equipped with 6 flush mounted thin foil thermocouples on its cold-side surface. Measurements were conducted with the 1.6$\mathrm{mm}$ thick liquid bismuth alloy layer between the top of the water-cooled plate and the bottom surface of the test sample. The measured effective thermal conductivities and their corresponding measurement uncertainties as a function of $T_{H}$ for chamber static pressures of $0.001,1,10$, and 100 torr are shown

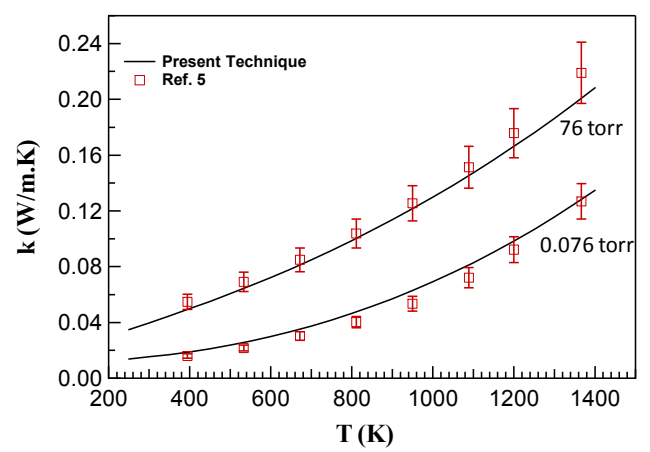

a)

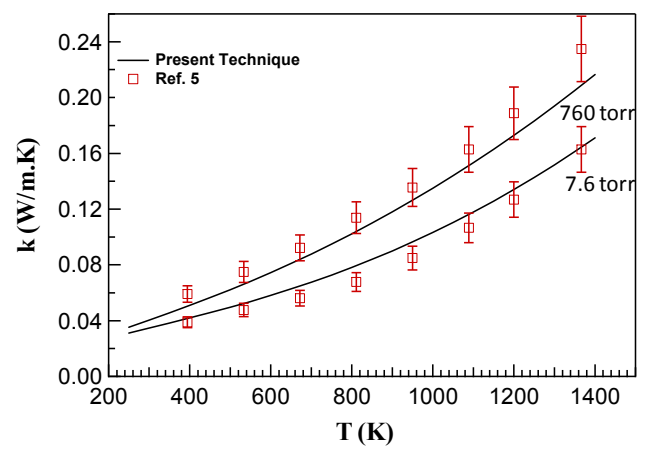

b)

Figure 13. Comparison of predicted thermal conductivity of LI-900 with published data in air at pressures of: a) 0.076 and 76 torr; b) 7.6 and 760 torr.

in Fig. 14. The average uncertainty for the effective thermal conductivity test data on the AETB- 8 tile for all test temperatures and pressures was $\pm 7.9 \%$. The average and standard deviations for all test data on this tile were 332.2 $\pm 9.6 \mathrm{~K}, 339 \pm 16.6 \mathrm{~K}$, and $6.8 \pm 8.1 \mathrm{~K}$, for $T_{C}, T_{B}$, and $T_{B}-T_{C}$, respectively. The relatively low value of $T_{B}-T_{C}$ implied that the use of the liquid bismuth alloy had effectively eliminated thermal contact resistance. The effective thermal conductivity data at 0.001 torr were used in conjunction with Eqs. (2) and (5) for estimating $k_{s r}$. The variation of predicted AETB-8 thermal conductivity with temperature at 0.001 torr is shown in Fig.15. The measured effective thermal conductivity data at higher pressures in nitrogen were used to estimate the characteristic length for gas conduction, which was determined to be $1.7 \times 10^{-4} \mathrm{~m}$. The comparison of predicted thermal conductivities with transient radiant step heating (TRSH) data in nitrogen gas is shown in Fig. 16 for static pressures of 0.76 and 7.6 torr. The reported TRSH data uncertainties of $\pm 10 \%$ are shown in the figure as error bars. The rms deviations between predictions and TRSH data were 13.9 , and $10 \%$ at pressures of 0.76 , and 7.6 torr, respectively. This further validated the present technique's methodology. Given the combined contribution of radiation and solid conduction at 0.001 torr, and the estimated gas conduction characteristic length, thermal conductivity data for AETB- 8 can be generated for any gaseous medium for any temperature between 300 and $1400 \mathrm{~K}$ and pressure between 0.001 and 760 torr. The predicted thermal conductivity of AETB- 8 as a function of temperature for various pressures in argon gas is shown in Fig. 17. 


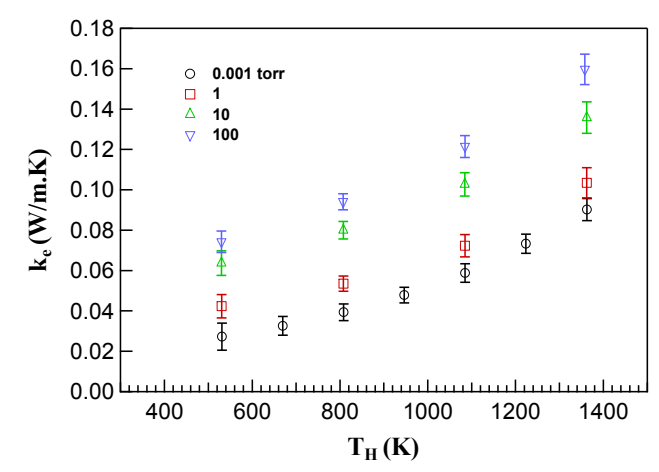

Figure 14. Variation of measured effective thermal conductivity with hot-side temperature at various pressures for AETB-8.

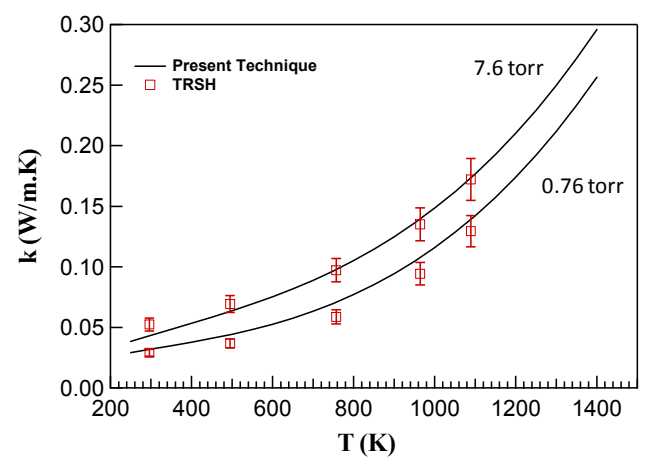

Figure 16. Comparison of predicted thermal conductivity of AETB-8 with transient radiant step heating (TRSH) data in nitrogen at pressures of 0.076 and 7.6 torr.

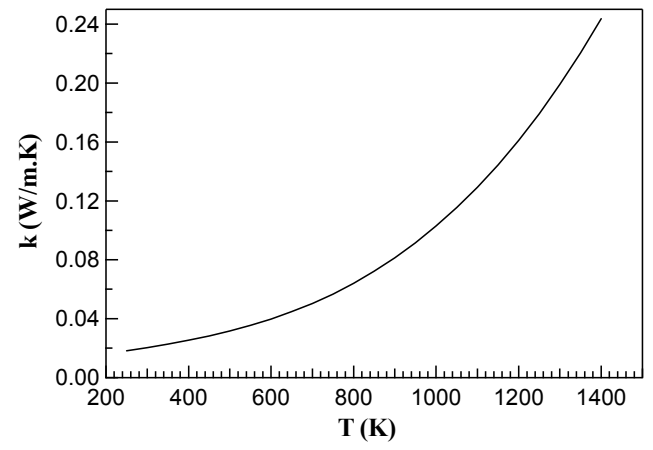

Figure 15. Variation of predicted thermal conductivity with temperature at 0.001 torr for AETB-8.

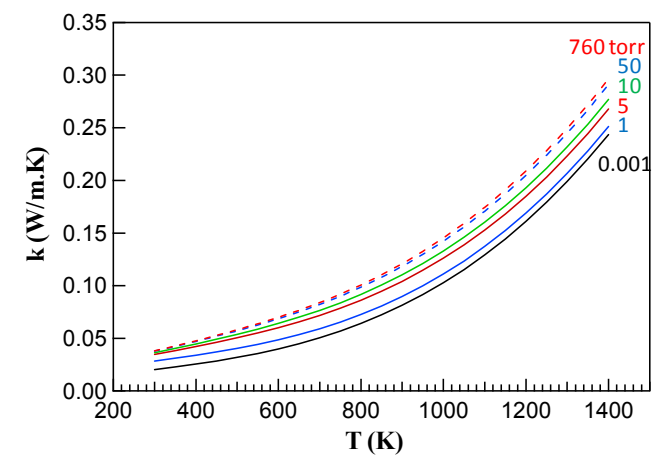

Figure 17. Predicted thermal conductivity of AETB-8 in argon at pressures of 0.001, 1, 5, 10, 50, and 760 torr.

\section{AETB-12}

The AETB-12 tile had a density of $187.8 \mathrm{~kg} / \mathrm{m}^{3}$ and was equipped with 3 uncoated flush mounted thin foil thermocouples on its cold side surface. Measurements were conducted with the 1.6-mm thick liquid bismuth alloy layer between the top of the water-cooled plate and the bottom of the test sample. The measured effective thermal conductivities as a function of $T_{H}$ for chamber static pressures of $0.001,1,10$, and 750 torr are shown in Fig. 18. The effective thermal conductivity measurement uncertainties for each data point are also shown in the figure. The average uncertainty for the effective thermal conductivity test data on the AETB-12 tile for all test temperatures and pressures was $\pm 7.3 \%$. The average and standard deviation for all test data were $332 \pm 9 \mathrm{~K}, 336 \pm$ $12.8 \mathrm{~K}$, and $4 \pm 5.1 \mathrm{~K}$, for $T_{C}, T_{B}$, and $T_{B}-T_{C}$, respectively. The effective thermal conductivity data at 0.001 torr were used in conjunction with Eqs. (2) and (5) for estimating $k_{s r}$, which is shown in Fig. 19. The corresponding AETB-8 thermal conductivity at 0.001 torr is also included in this figure. At temperatures above $800 \mathrm{~K}$, AETB- 12 has a lower thermal conductivity compared to AETB-8. As density increases from 133.6 to $187.8 \mathrm{~kg} / \mathrm{m}^{3}$ the contribution of solid conduction increases, but the contribution of radiation heat transfer decreases. At lower temperatures, $\mathrm{T}<800 \mathrm{~K}$, solid

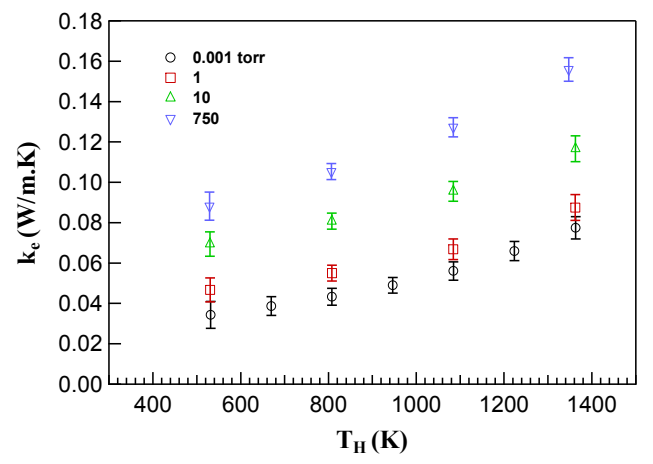

Figure 18. Variation of measured effective thermal conductivity with hot-side temperature at various pressures for AETB-12. 
higher density of AETB-12 causes an overall increase in thermal conductivity compared to AETB-8. At higher temperatures, $\mathrm{T}>800 \mathrm{~K}$, radiation is the dominant mode of heat transfer, so the higher density of AETB-12 causes an overall decrease in thermal conductivity compared to AETB-8. The thermal conductivity of AETB-12 is 5.9 to 23.2\% lower than AETB-8 between 800 and $1400 \mathrm{~K}$.

The measured effective thermal conductivity data in nitrogen at higher pressures were used to estimate the characteristic length for gas conduction, which was determined to be $1.23 \times 10^{-4} \mathrm{~m}$, slightly lower than the AETB-8 gas conduction characteristic length. The comparison of predicted thermal conductivities with TRSH data in nitrogen gas is shown in Fig. 20 for static pressures of 7.6 and 76 torr. The rms deviations between predictions and TRSH data were 12.6 , and $7.4 \%$ at pressures of 0.76 , and 7.6 torr, respectively. This further validated the present technique's methodology. The predicted thermal conductivity of AETB12 as a function of temperature for various pressures in dry air is shown in Fig. 21.

\section{E. Summary}

Overall, it was shown that thermal contact resistance can be significant on the rigid insulation test sample cold-side surface. Obtaining perfectly flat surfaces on ceramic tiles is not easily achievable, and even an initially flat surface may bow due to thermal expansion differences caused by thermal gradients through the sample. Utilizing a thin layer of liquid bismuth alloy between the sample cold-side surface and the test setup water-cooled plate proved to be an efficient method for effectively eliminating thermal contact resistance in the test setup. A methodology was investigated for estimating thermal conductivity of rigid insulations in various gaseous environments for the temperature range of $300-1400 \mathrm{~K}$ and pressure range of 0.001 to 760 torr. The method consisted of using thermal data at 0.001 torr to model the combined contribution of solid conduction and radiation heat transfer. Data at higher pressures in nitrogen were then used to estimate the gas conduction characteristic length. These data can then be used to estimate thermal conductivity of the insulation at various temperatures and pressures in various gaseous environments. The methodology was validated by comparing estimated thermal conductivity of LI-900 with published data. The methodology was also applied to AETB-8 and AETB-12 tiles, and data were compared with limited TRSH data. It should be noted that the methodology used here could have also been used in conjunction with standard thermal conductivity data (with small temperature differences maintained across sample thickness) instead of the effective thermal conductivity data with large temperature differences.

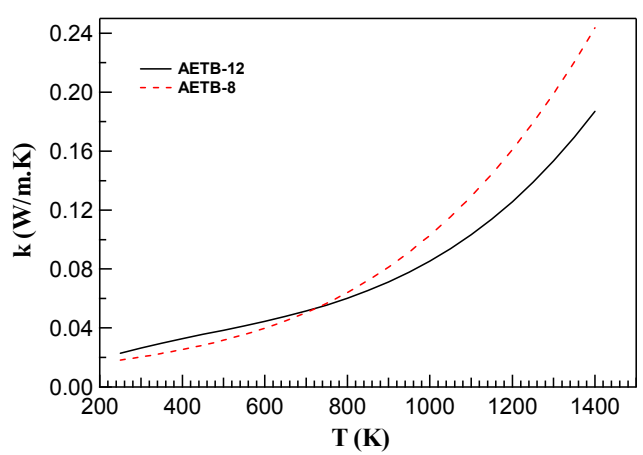

Figure 19. Variation of predicted thermal conductivity with temperature at 0.001 torr for AETB-12 and AETB-8.

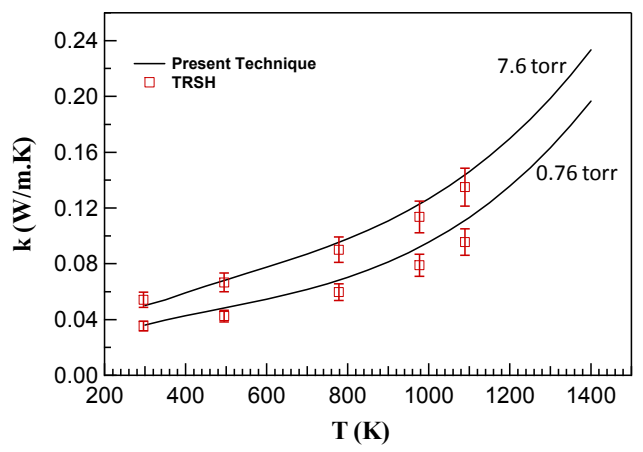

Figure 20. Comparison of predicted thermal conductivity of AETB-12 with transient radiant step heating (TRSH) data in nitrogen at pressures of 0.76 and 7.6 torr.

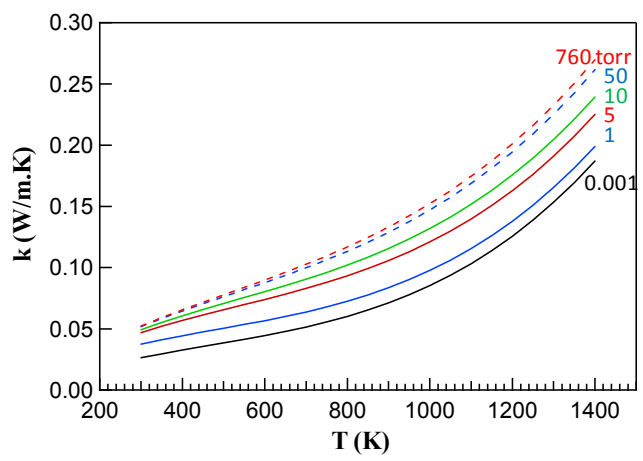

Figure 21. Predicted thermal conductivity of AETB-12 in dry air at pressures of $0.001,1,5,10$, 50 , and 760 torr.

\section{Concluding Remarks}

Effective thermal conductivity of three rigid fibrous insulations was measured in the NASA LaRC thermalvacuum testing apparatus. Thermal contact resistance between the bottom surface of LI-900 tile and the test setup water-cooled plate was investigated. Significant temperature differences were measured between the sample cold- 
side surface and the adjoining water-cooled plate. The temperature differences increased with decreasing test chamber pressure and increasing sample hot-side temperature (increasing heat flux). Temperature differences varied between 13 and $201 \mathrm{~K}$. The associated thermal contact resistance decreased with increasing chamber test pressure, and was not sensitive to sample hot-side temperatures, except at the lowest pressure of 0.001 torr. At this pressure thermal contact resistance was inversely proportional to sample hot-side temperature.

A technique for eliminating thermal contact resistance was investigated. The technique consisted of using a thin layer of liquid bismuth alloy, maintained at approximately $330 \mathrm{~K}$, between the sample sold-side surface and the test setup water-cooled plate. The resulting temperature differences between the sample cold-side surface and watercooled plate varied between 0.9 and $8.3 \mathrm{~K}$, which were the normal temperature drops through liquid bismuth due to conduction heat transfer, thus effectively eliminating thermal contact resistance.

A methodology was investigated for estimating relevant parameters needed for modeling combined radiation and conduction heat transfer in rigid fibrous insulations. Effective thermal conductivity measurements in a vacuum were used to estimate the combined contribution of solid conduction and radiation heat transfer. Effective thermal conductivity measurements at higher pressures were used to estimate the gas conduction characteristic length. With this information, thermal conductivity data of the rigid tiles can then be generated in various gaseous media at various temperatures $(300-1400 \mathrm{~K})$ and static pressures $(0.001-760$ torr). Estimated thermal conductivity data on an LI-900 tile were compared with published data. The overall rms deviation between predicted and published data was $10.5 \%$. Given the $10 \%$ uncertainty in published data, this close agreement validated the methodology used in the present study. The same methodology was also applied to AETB-8 and AETB-12 tiles, and predicted data were compared with transient radiant step heating technique data.

\section{Acknowledgments}

The authors wish to express their gratitude to the following individuals for their invaluable contributions: Mr. Wayne Geouge, NASA Langley Research Center, for fabrication and instrumentation of steady-state test setup and test samples; Jozef Gembarovic, TPRL Inc, for providing transient step heating technique thermal conductivity

measurements. This work was funded through NASA Fundamental Aeronautics Hypersonic Materials and Structures Project.

\section{References}

1. Daryabeigi, K., Cunnington, G. R., Miller, S. D., and Knutson, J. R., "Combined Heat Transfer in HighPorosity High-Temperature Fibrous Insulations: Theory and Experimental Validation," AIAA Paper 20104660, June 2010.

2. Banas, R. P., and Cunnington, G. R., "Determination of Effective Thermal Conductivity for the Space Shuttle Orbiter's Reusable Surface Insulation (RSI)," AIAA Paper 74-730, July 1974.

3. Williams, S. D., and Curry, D. M., "Prediction of Rigid Silica Based Insulation Conductivity," NASA TP3276, January 1993.

4. Verschoor, J. D., Greebler, P., and Manville, N. J., "Heat Transfer by Gas Conduction and Radiation in Fibrous Insulations," Transactions of the American Society of Mechanical Engineers, Vol. 74, No. 8, 1952, pp. 961-968.

5. Lee, S. C., and Cunnington, G. R., "Heat transfer in Fibrous Insulations: Comparison of Theory and Experiment," Journal of Thermophysics and Heat Transfer, Vol. 12, No. 3, 1998, pp. 297-303.

6. Kennard, E. H., Kinetic Theory of Gases, McGraw-Hill, New York, 1938, pp. 163-179, 290-318.

7. Raed, K. and Gross, U., "Review on Gas Thermal Conductivity in Porous Materials and Knudsen Effect," Thermal Conductivity, edited by J.R. Koenig and H. Ban, Vol. 29, DEStech Publications Inc., Lancaster, PA, 2008, pp. 356-373.

8. Lee, S. C., and Cunnington, G. R, "Theoretical Models for Radiative Transfer in Fibrous Media," Annual Review of Heat Transfer, Edited by C. L. Tien, Vol. 9, Begell House, New York, 1998, pp. 159-212.

9. Roux, J. A., and Smith, A. M., "Combined Conductive and Radiative Heat Transfer in an Absorbing and Scattering Medium," American Institute of Chemical Engineers and American Society of Mechanical Engineers Heat Transfer Conference, Paper 77-HT-50, Aug. 1977. 
10. Matthews, L. K., Viskanta, R., and Incropera, F. P., "Combined Conduction and Radiation Heat Transfer in Porous Materials Heated by Intense Solar Radiation," Journal of Solar Energy Engineering, Vol. 107, No. 1, 1985, pp. 29-34.

11. Lee, S. C., "Radiative Transfer through a Fibrous Medium: Allowance for Fiber Orientation," Journal of Quantitative Spectroscopy and Radiative Transfer, Vol. 36, No. 3, 1986, pp. 253-263.

12. Lee, S. C., "Scattering Phase Function for Fibrous Media," International Journal of Heat and Mass Transfer, Vol. 33, No. 10, 1990, pp. 2183-2190.

13. Goldstein, H. E., Leiser, D. B., Smith, M., and Stewart, D. A., "Opacified Silica Reusable Surface Insulation (RSI) for Thermal Protection of the Space Shuttle Orbiter," Thermal Conductivity, edited by V. V. Mirkovich, Vol. 15, Plenum Press, New York, NY, 1977, pp. 335-341.

14. Daryabeigi, K., "Effective Thermal Conductivity of High Temperature Insulations for Reusable Launch Vehicles," NASA TM-1999-208972, February 1999.

15. Daryabeigi, K., "Analysis and Testing of High Temperature Fibrous Insulation for Reusable Launch Vehicles," AIAA Paper 99-1044, January 1999.

16. Gembarovic, J., and Taylor, R. E, "A Method for Thermal Diffusivity Determination of Thermal Insulators," International Journal of Thermophysics, Vol. 28, No. 6, 2007, pp. 2164-2175.

17. Touloukian, Y. S., Liley, P. E., and Saxena, S. C., Thermal Conductivity, Nonmetallic Liquids and Gases, Vol. 3, Thermophysical Properties of Matter, IFI/Plenum, New York-Washington, 1970.

18. Touloukian, Y. S., and Makita, T., Specific Heat, Nonmetallic Liquids and Gases, Vol. 6, Thermophysical Properties of Matter, IFI/Plenum, New York-Washington, 1970.

19. Touloukian, Y. S., Saxena, S. C., and Hestermans, Viscosity, Vol. 11, Thermophysical Properties of Matter, IFI/Plenum, New York-Washington, 1970. 